\title{
Existence and Uniqueness of Solutions of Integer Order Differential Equations with Non-Instantaneous Impulses
}

\author{
WenJing Zheng \\ College of Science, University of Shanghai for Science and Technology, Shanghai, P. R. China \\ Email: jokerzheng0201@163.com
}

\begin{abstract}
We study the existence and uniqueness of solutions for a class of integer order differential equations with non-instantaneous. Firstly, the differential boundary value problem is transformed into an equivalent integral equation problem, and then the existence results of the solution and the sufficient conditions for the existence of the solutions are obtained by using Schauder fixed point theory. The uniqueness theorem of the solution is established by using contraction mapping principle.
\end{abstract}

Keywords: non-instantaneous impulsive, Caputo derivative, contraction mapping principle, Schauder fixed point theorem

\section{Introduction}

Impulsive differential equations are a generalization of differential equations. With the development of differential equations, differential equations have been widely used in the modeling of different physical and natural science fields such as fluid mechanics, chemistry, control systems, and heat conduction. Many practical problems are under development. There are rapid changes in certain stages of the process, which are called impulses. There are two main types of pulses, one is instantaneous impulses, and the other is non- instantaneous impulses.

In instantaneous impulses, the duration of change is negligible compared to the duration of the entire evolution (such as shocks and natural disasters). Professor Mil'man and Professor Myshkis first proposed the transient pulse differential equation in the 1960s. Since then, many mathematicians have devoted themselves to the research of instantaneous differential equations, and have obtained the profound conclusions about the existence, uniqueness and stability of the solutions of instantaneous impulsive differential equations, branch theory, and dynamic systems with impulses. There are many literatures and works on the study of the boundary value problem of instantaneous impulsive differential equations, see literature [1]-[3], and the theoretical results are also increasingly perfect.

In non-instantaneous impulses, the duration of change stays within a limited time interval. This phenomenon is common in all fields of modern science and technology. For example, in clinical treatment, the process of injecting a drug into the body can be regarded as a non-instantaneous pulse therapy behavior. Drug absorption and action in the human body is a continuous and gradual process, which can be expressed by a differential equation. After the drug is absorbed and metabolized in the human body, the body's own metabolic changes can be determined by another function. Control, because this phenomenon can more deeply and accurately reflect the changing rules of things, it has attracted extensive attention from a large number of scholars, which has led mathematicians to study non-instantaneous impulsive differential equations, see references [4]-[9].Non-transient impulsive differential equation is a very good mathematical model that comes from both practice and practice, so it has very important theoretical basis and practical value. This paper studies a class of integer order differential equations with non-instantaneous impulses boundary value problems:

$$
\left\{\begin{array}{l}
u^{\prime}(t)=f(t, u(t)), t \in\left(s_{k}, t_{k+1}\right], k=0,1,2 \cdots, m, \\
u^{\prime}(t)=g(t, u(t)), t \in\left(t_{k}, s_{k}\right], k=1,2 \cdots, m, \\
\left.\Delta u\right|_{t=t_{k}}=M_{k}\left(t_{k}, u\left(t_{k}\right)\right),\left.\Delta u\right|_{t=s_{k}}=Q_{k}\left(s_{k}, u\left(s_{k}\right)\right), \\
a u(0)+b u(1)=0,
\end{array}\right.
$$


where $0=s_{0}<t_{1}<s_{1}<t_{2}<\cdots<s_{m}<t_{m+1}=1, \quad J=[0,1], a, b \in R, u\left(t_{k}^{+}\right), u\left(t_{k}^{-}\right), u\left(s_{k}^{+}\right), u\left(s_{k}^{-}\right)$exist $f, g, M_{k}, Q_{k}: J \times \mathbb{R} \rightarrow \mathbb{R}$ are continuous, $u\left(t_{k}\right)=u\left(t_{k}^{-}\right), u\left(s_{k}\right)=u\left(s_{k}^{-}\right) .\left.\quad \Delta u\right|_{t=t_{k}}=\Delta u\left(t_{k}\right)=u\left(t_{k}^{+}\right)-u\left(t_{k}^{-}\right)$, $\left.\Delta u\right|_{t=s_{k}}=\Delta u\left(s_{k}\right)=u\left(s_{k}^{+}\right)-u\left(s_{k}^{-}\right), k=1,2, \cdots, m$.

\section{Paper Preparation}

Define $\quad$ space $\quad P C(J, \mathbb{R}):=\left\{u: J \rightarrow \mathbb{R} \mid u \in C\left(J^{\prime}, \mathbb{R}\right), u\left(t_{k}^{-}\right), u\left(t_{k}^{+}\right)\right.$exist, $\left.u\left(t_{k}\right)=u\left(t_{k}^{-}\right), k=1,2, \cdots, m\right\} \quad, \quad$ let $\|u\|_{P C}=\sup _{t \in[0,1]}|u(t)|$ and $P C(J, \mathbb{R})$ is a Banach Space.

Definition 2.1 Let $u \in P C(J, \mathbb{R})$, if $u$ meet various conditions in (1), then we call it a solution to the boundary value problem (1).

Lemma 2.1 For any given $y(t) \in C[0,1], h(t) \in C[0,1]$, the following boundary value problem

has the following form solution

$$
\left\{\begin{array}{l}
u^{\prime}(t)=y(t), \quad t \in\left(s_{k}, t_{k+1}\right], \quad k=0,1,2 \cdots, m, \\
u^{\prime}(t)=h(t), t \in\left(t_{k}, s_{k}\right], \quad k=1,2 \cdots, m, \\
\left.\Delta u\right|_{t=t_{k}}=m_{k}\left(t_{k}, u\left(t_{k}\right)\right),\left.\Delta u\right|_{t=s_{k}}=q_{k}\left(s_{k}, u\left(s_{k}\right)\right), \\
a u(0)+b u(1)=0,
\end{array}\right.
$$

$$
u(t)=\left\{\begin{array}{l}
\sum_{i=1}^{k}\left(\int_{t_{i}}^{s_{i}} h(s) \mathrm{d} s+\int_{s_{i-1}}^{t_{i}} y(s) \mathrm{d} s+m_{i}+q_{i}\right)-\frac{a}{a+b} \int_{s_{m}}^{1} f(s) \mathrm{d} s t \in\left[0, t_{1}\right], \\
\int_{s_{k}}^{t} f(s) \mathrm{d} s+\frac{a}{a+b} \sum_{i=1}^{k}\left(\int_{t_{i}}^{s_{i}} h(s) \mathrm{d} s+\int_{s_{i-1}}^{t_{i}} y(s) \mathrm{d} s+m_{i}+q_{i}\right) \\
\quad-\frac{a}{a+b} \int_{s_{m}}^{1} f(s) \mathrm{d} s t \in\left[0, t_{1}\right], t \in\left(s_{k}, t_{k+1}\right], k=1,2, \cdots, m \\
\int_{t_{k}}^{t} g(s) \mathrm{d} s+\frac{a}{a+b} \sum_{i=1}^{k-1}\left(\int_{t_{i}}^{s_{i}} h(s) \mathrm{d} s+\int_{s_{i-1}}^{t_{i}} y(s) \mathrm{d} s+m_{i}+q_{i}\right) \\
\quad-\frac{a}{a+b}\left(\int_{t_{k}}^{s_{k}} h(s) \mathrm{d} s+\int_{s_{k-1}}^{t_{k}} y(s) \mathrm{d} s+m_{k}+q_{k}\right)+m_{k}+\int_{s_{k-1}}^{t_{k}} f(s) \mathrm{d} s, \quad t \in\left(t_{k}, s_{k}\right], k=1,2, \cdots, m
\end{array}\right.
$$

Proof. When $t \in\left[0, t_{1}\right]$, from $u^{\prime}(t)=y(t)$ we have

$$
u(t)=\int_{0}^{t} y(s) \mathrm{d} s+c_{0}, u\left(t_{1}^{-}\right)=\int_{0}^{t_{1}} y(s) \mathrm{d} s+c_{0} .
$$

When $t \in\left(t_{1}, s_{1}\right]$, consider Cauchy problem

$$
\left\{\begin{array}{l}
u^{\prime}(t)=h(t), t \in\left(t_{1}, s_{1}\right], \\
\left.\Delta u\right|_{t=t_{1}}=m_{1},\left.\Delta u\right|_{t=s_{1}}=q_{1},
\end{array}\right.
$$

from

$$
u^{\prime}(t)=h(t), u(t)=\int_{t_{1}}^{t} h(\mathrm{~s}) \mathrm{d} s+d_{1}, u\left(s_{1}^{-}\right)=\int_{t_{1}}^{s_{1}} h(\mathrm{~s}) \mathrm{d} s+d_{1}
$$

we have $d_{1}=\int_{0}^{t_{1}} y(s) \mathrm{d} s+m_{1}+c_{0}$, and

$$
u(t)=\int_{t_{1}}^{t} h(\mathrm{~s}) \mathrm{d} s+\int_{0}^{t_{1}} y(s) \mathrm{d} s+m_{1}+c_{0},
$$

thus $u\left(s_{1}^{-}\right)=\int_{t_{1}}^{s_{1}} h(\mathrm{~s}) \mathrm{d} s+\int_{0}^{t_{1}} y(s) \mathrm{d} s+m_{1}+c_{0}$.

When $t \in\left(s_{1}, t_{2}\right]$, consider Cauchy problem

from $u^{\prime}(t)=y(t)$ we have

$$
\left\{\begin{array}{l}
u^{\prime}(t)=y(t), \\
\left.\Delta u\right|_{t=t_{2}}=m_{1},\left.\Delta u\right|_{t=s_{1}}=q_{1},
\end{array}\right.
$$




$$
u(t)=\int_{s_{1}}^{t} y(s) \mathrm{d} s+c_{1}, u\left(s_{1}^{+}\right)=c_{1}=\int_{0}^{t_{1}} y(\mathrm{~s}) \mathrm{d} s+\int_{t_{1}}^{s_{1}} h(\mathrm{~s}) \mathrm{d} s+m_{1}+q_{1}+c_{0},
$$

Now consider the general situation of the time when $k=1,2,3, \cdots m$,

When $t \in\left(s_{k-1}, t_{k}\right]$, from $u^{\prime}(t)=y(t)$ we have

$$
u(t)=\int_{s_{k-1}}^{t} y(\mathrm{~s}) \mathrm{d} s+c_{k-1}, u\left(t_{k}^{-}\right)=\int_{s_{k-1}}^{t_{k}} y(\mathrm{~s}) \mathrm{d} s+c_{k-1},
$$

When $t \in\left(t_{k}, s_{k}\right]$,

$$
\left\{\begin{array}{l}
u^{\prime}(t)=h(t), \\
\left.\Delta u\right|_{t=t_{k}}=m_{k},\left.\Delta u\right|_{t=s_{k}}=q_{k},
\end{array}\right.
$$

We have

$$
u(t)=\int_{t_{k}}^{t} h(s) \mathrm{d} s+d_{k},
$$

from $\left.\Delta u\right|_{t=t_{k}}=m_{k}$ we have

$$
\begin{gathered}
u\left(t_{k}^{+}\right)=d_{k}=\int_{s_{k-1}}^{t_{k}} y(s) \mathrm{d} s+m_{k}+c_{k-1}, \\
u\left(s_{k}^{-}\right)=\int_{s_{k-1}}^{t_{k}} y(s) \mathrm{d} s+\int_{t_{k}}^{s_{k}} h(\mathrm{~s}) \mathrm{d} s+m_{k}+c_{k-1}, \\
u\left(s_{k}^{+}\right)=c_{k}=\int_{s_{k-1}}^{t_{k}} y(s) \mathrm{d} s+\int_{t_{k}}^{s_{k}} h(s) \mathrm{d} s+m_{k}+q_{k}+c_{k-1}, \\
c_{k}-c_{k-1}=\int_{s_{k-1}}^{t_{k}} y(s) \mathrm{d} s+\int_{t_{k}}^{s_{k}} h(s) \mathrm{d} s+m_{k}+q_{k},
\end{gathered}
$$

we have

$$
c_{k}=c_{0}+\sum_{i=1}^{k} \int_{s_{i-1}}^{t_{i}} y(s) \mathrm{d} s+\int_{t_{i}}^{s_{i}} h(s) \mathrm{d} s+m_{i}+q_{i}
$$

so

$$
d_{k}=c_{0}+\int_{s_{k-1}}^{t_{k}} y(s) \mathrm{d} s+m_{k}+\sum_{i=1}^{k-1}\left(\int_{s_{i-1}}^{t_{i}} y(\mathrm{~s}) \mathrm{d} s+\int_{t_{i}}^{s_{i}} h(\mathrm{~s}) \mathrm{d} s+m_{i}+q_{i}\right) .
$$

Above all, when $t \in\left(s_{k}, t_{k+1}\right]$,

$$
u(t)=\int_{s_{k}}^{t} y(s) \mathrm{d} s+\mathrm{c}_{k}=\int_{s_{k}}^{t} y(s) \mathrm{d} s+\sum_{i=1}^{k}\left(\int_{s_{i-1}}^{t_{i}} y(s) \mathrm{d} s+\int_{t_{i}}^{s_{i}} h(\mathrm{~s}) \mathrm{d} s+m_{i}+q_{i}\right)+c_{0} .
$$

when $t \in\left(t_{k}, s_{k}\right]$,

$$
u(t)=\int_{t_{k}}^{t} h(s) \mathrm{d} s+d_{k}=\int_{t_{k}}^{t} h(s) \mathrm{d} s+\int_{s_{k-1}}^{t_{k}} y(s) \mathrm{d} s+m_{k}+\sum_{i=1}^{k-1}\left(\int_{s_{i-1}}^{t_{i}} y(s) \mathrm{d} s+\int_{t_{i}}^{s_{i}} h(s) \mathrm{d} s+m_{i}+q_{i}\right)+c_{0} .
$$

from $a u(0)+b u(1)=0$, we have

$$
\begin{gathered}
u(0)=c_{0}, u(1)=\int_{s_{m}}^{1} y(s) \mathrm{d} s+\sum_{i=1}^{m}\left(\int_{s_{i-1}}^{t_{i}} y(s) \mathrm{d} s+\int_{t_{i}}^{s_{i}} h(s) \mathrm{d} s+m_{i}+q_{i}\right)+c_{0}, \\
a u(0)+b u(1)=a c_{0}+b\left(\int_{s_{m}}^{1} y(s) \mathrm{d} s+\sum_{i=1}^{m}\left(\int_{s_{i-1}}^{t_{i}} y(s) \mathrm{d} s+\int_{t_{i}}^{s_{i}} h(s) \mathrm{d} s+m_{i}+q_{i}\right)+c_{0}\right)=0,
\end{gathered}
$$

thus

$$
\begin{gathered}
c_{0}=\frac{-b}{(a+b)}\left(\int_{s_{m}}^{1} y(s) \mathrm{d} s+\sum_{i=1}^{m}\left(\int_{s_{i-1}}^{t_{i}} y(s) \mathrm{d} s+\int_{t_{i}}^{s_{i}} h(s) \mathrm{d} s+m_{i}+q_{i}\right)\right) \\
=-\frac{b}{(a+b)}\left(\sum_{i=1}^{m+1} \int_{s_{i-1}}^{t_{i}} y(s) \mathrm{d} s+\sum_{i=1}^{m}\left(\int_{t_{i}}^{s_{i}} h(s) \mathrm{d} s+m_{i}+q_{i}\right)\right), \\
c_{k}=\sum_{i=1}^{k}\left(\int_{s_{i-1}}^{t_{i}} y(\mathrm{~s}) \mathrm{d} s+\int_{t_{i}}^{s_{i}} h(\mathrm{~s}) \mathrm{d} s+m_{i}+q_{i}\right)-\frac{b}{(a+b)}\left(\sum_{i=1}^{m+1} \int_{s_{i-1}}^{t_{i}} y(s) \mathrm{d} s+\sum_{i=1}^{m}\left(\int_{t_{i}}^{s_{i}} h(\mathrm{~s}) \mathrm{d} s+m_{i}+q_{i}\right)\right),
\end{gathered}
$$




$$
\begin{aligned}
d_{k}= & c_{0}+\int_{s_{k-1}}^{t_{k}} y(\mathrm{~s}) \mathrm{d} s+m_{k}+\sum_{i=1}^{k-1}\left(\int_{s_{i-1}}^{t_{i}} y(\mathrm{~s}) \mathrm{d} s+\int_{t_{i}}^{s_{i}} h(\mathrm{~s}) \mathrm{d} s+m_{i}+q_{i}\right) \\
= & \int_{s_{k-1}}^{t_{k}} y(\mathrm{~s}) \mathrm{d} s+m_{k}+\sum_{i=1}^{k-1}\left(\int_{s_{i-1}}^{t_{i}} y(\mathrm{~s}) \mathrm{d} s+\int_{t_{i}}^{s_{i}} h(\mathrm{~s}) \mathrm{d} s+m_{i}+q_{i}\right) \\
& -\frac{b}{(a+b)}\left(\sum_{i=1}^{m+1} \int_{s_{i-1}}^{t_{i}} y(s) \mathrm{d} s+\sum_{i=1}^{m}\left(\int_{t_{i}}^{s_{i}} h(\mathrm{~s}) \mathrm{d} s+m_{i}+q_{i}\right)\right) .
\end{aligned}
$$

Above all, when $t \in\left(s_{k}, t_{k+1}\right]$,

$$
\begin{aligned}
u(t)= & \int_{s_{k}}^{t} y(\mathrm{~s}) \mathrm{d} s+\mathrm{c}_{k} \\
= & \int_{s_{k}}^{t} y(\mathrm{~s}) \mathrm{d} s+\sum_{i=1}^{k}\left(\int_{s_{i-1}}^{t_{i}} y(\mathrm{~s}) \mathrm{d} s+\int_{t_{i}}^{s_{i}} h(\mathrm{~s}) \mathrm{d} s+m_{i}+q_{i}\right)-\frac{b}{(a+b)}\left(\sum_{i=1}^{m+1} \int_{s_{i-1}}^{t_{i}} y(s) \mathrm{d} s+\sum_{i=1}^{m}\left(\int_{t_{i}}^{s_{i}} h(\mathrm{~s}) \mathrm{d} s+m_{i}+q_{i}\right)\right) \\
= & \int_{s_{k}}^{t} y(\mathrm{~s}) \mathrm{d} s+\sum_{\mathrm{i}=1}^{k} \int_{s_{i-1}}^{t_{i}} y(\mathrm{~s}) \mathrm{d} s-\frac{b}{(a+b)} \sum_{i=1}^{m+1} \int_{s_{i-1}}^{t_{i}} y(s) \mathrm{d} s \\
& +\sum_{i=1}^{k} \int_{t_{i}}^{s_{i}} h(\mathrm{~s}) \mathrm{d} s-\frac{b}{(a+b)} \sum_{i=1}^{m} \int_{t_{i}}^{s_{i}} h(\mathrm{~s}) \mathrm{d} s+\sum_{i=1}^{k}\left(m_{i}+q_{i}\right)-\frac{b}{(a+b)} \sum_{i=1}^{m}\left(m_{i}+q_{i}\right)
\end{aligned}
$$

when $t \in\left(t_{k}, s_{k}\right]$,

$$
\begin{aligned}
u(t)= & \int_{t_{k}}^{t} h(\mathrm{~s}) \mathrm{d} s+d_{k}=\int_{t_{k}}^{t} h(\mathrm{~s}) \mathrm{d} s+\int_{s_{k-1}}^{t_{k}} y(\mathrm{~s}) \mathrm{d} s+m_{k}+\sum_{i=1}^{k-1}\left(\int_{s_{i-1}}^{t_{i}} y(\mathrm{~s}) \mathrm{d} s+\int_{t_{i}}^{s_{i}} h(\mathrm{~s}) \mathrm{d} s+m_{i}+q_{i}\right)+c_{0} \\
= & \int_{t_{k}}^{t} h(\mathrm{~s}) \mathrm{d} s+\int_{s_{k-1}}^{t_{k}} y(\mathrm{~s}) \mathrm{d} s+m_{k}+\sum_{i=1}^{k-1}\left(\int_{s_{i-1}}^{t_{i}} y(\mathrm{~s}) \mathrm{d} s+\int_{t_{i}}^{s_{i}} h(\mathrm{~s}) \mathrm{d} s+m_{i}+q_{i}\right) \\
& -\frac{b}{(a+b)}\left(\sum_{i=1}^{m+1} \int_{s_{i-1}}^{t_{i}} y(s) \mathrm{d} s+\sum_{i=1}^{m}\left(\int_{t_{i}}^{s_{i}} h(\mathrm{~s}) \mathrm{d} s+m_{i}+q_{i}\right)\right) \\
= & \int_{s_{k-1}}^{t_{k}} y(\mathrm{~s}) \mathrm{d} s+\sum_{i=1}^{k-1} \int_{s_{i-1}}^{t_{i}} y(\mathrm{~s}) \mathrm{d} s-\frac{b}{(a+b)} \sum_{i=1}^{m+1} \int_{s_{i-1}}^{t_{i}} y(s) \mathrm{d} s+\int_{t_{k}}^{t} h(\mathrm{~s}) \mathrm{d} s+\sum_{i=1}^{k-1} \int_{t_{i}}^{s_{i}} h(\mathrm{~s}) \mathrm{d} s-\frac{b}{(a+b)} \sum_{i=1}^{m} \int_{t_{i}}^{s_{i}} h(\mathrm{~s}) \mathrm{d} s \\
& +m_{k}+\sum_{i=1}^{k-1}\left(m_{i}+q_{i}\right)-\frac{b}{(a+b)} \sum_{i=1}^{m}\left(m_{i}+q_{i}\right) .
\end{aligned}
$$

Let

$$
\begin{aligned}
\chi(x, y, z)= \begin{cases}1, & x \leq z \leq y \\
0, & \text { other. }\end{cases} \\
W_{1}(t, s)=\left\{\begin{array}{l}
\chi(0, t, s)-\frac{b}{(a+b)} \sum_{i=1}^{m+1} \chi\left(s_{i-1}, t_{i}, s\right), 0 \leq t \leq t_{1}, 0 \leq s \leq 1, \\
\chi\left(s_{k}, t, s\right)+\sum_{i=1}^{k} \chi\left(s_{i-1}, t_{i}, s\right)-\frac{b}{(a+b)} \sum_{i=1}^{m+1} \chi\left(s_{i-1}, t_{i}, s\right), s_{k}<t \leq t_{k+1}, 0 \leq s \leq 1, k=1,2, \cdots, m, \\
\sum_{i=1}^{k} \chi\left(s_{i-1}, t_{i}, s\right)-\frac{b}{(a+b)} \sum_{i=1}^{m+1} \chi\left(s_{i-1}, t_{i}, s\right), t_{k}<t \leq s_{k}, 0 \leq s \leq 1, k=1,2, \cdots, m,
\end{array}\right. \\
W_{2}(t, s)=\left\{\begin{array}{l}
-\frac{b}{(a+b)} \sum_{i=1}^{m} \chi\left(t_{i}, s_{i}, s\right), 0 \leq t \leq t_{1}, 0 \leq s \leq 1, \\
\sum_{i=1}^{k} \chi\left(t_{i}, s_{i}, s\right)-\frac{b}{(a+b)} \sum_{i=1}^{m} \chi\left(t_{i}, s_{i}, s\right), s_{k}<t \leq t_{k+1}, 0 \leq s \leq 1, k=1,2, \cdots, m, \\
\chi\left(t_{k}, t, s\right)+\sum_{i=1}^{k-1} \chi\left(t_{i}, s_{i}, s\right)-\frac{b}{(a+b)} \sum_{i=1}^{m} \chi\left(t_{i}, s_{i}, s\right), t_{k}<t \leq s_{k}, 0 \leq s \leq 1, k=1,2,3, \cdots, m .
\end{array}\right.
\end{aligned}
$$

Definition 2.2 Let operator $T, A, B, G: P C(J, \mathbb{R}) \rightarrow P C(J, \mathbb{R})$,

$$
A u(t)=\int_{0}^{1} W_{1}(t, s) f(s, u(s)) \mathrm{d} s, B u(t)=\int_{0}^{1} W_{2}(t, s) g(s, u(s)) \mathrm{d} s,
$$




$$
G u(t)=\left\{\begin{array}{c}
-\frac{b}{(a+b)} \sum_{i=1}^{m}\left(M_{i}\left(t_{i}, u\left(t_{i}\right)\right)+Q_{i}\left(t_{i}, u\left(t_{i}\right)\right)\right), t \in\left[0, t_{1}\right], \\
\sum_{i=1}^{k}\left(M_{i}\left(t_{i}, u\left(t_{i}\right)\right)+Q_{i}\left(t_{i}, u\left(t_{i}\right)\right)-\frac{b}{(a+b)} \sum_{i=1}^{m}\left(M_{i}\left(t_{i}, u\left(t_{i}\right)\right)+Q_{i}\left(t_{i}, u\left(t_{i}\right)\right),\right.\right. \\
t \in\left(s_{k}, t_{k+1}\right], k=1,2, \cdots, m, \\
M_{k}\left(t_{i}, u\left(t_{i}\right)\right)+\sum_{i=1}^{k-1}\left(M_{i}\left(t_{i}, u\left(t_{i}\right)\right)+Q_{i}\left(t_{i}, u\left(t_{i}\right)\right)\right. \\
-\frac{b}{(a+b)} \sum_{i=1}^{m}\left(M_{i}\left(t_{i}, u\left(t_{i}\right)\right)+Q_{i}\left(t_{i}, u\left(t_{i}\right)\right), t \in\left(t_{k}, \mathrm{~s}_{k}\right], k=1,2, \cdots, m,\right. \\
T u(t)=A u(t)+B u(t)+G u(t)
\end{array}\right.
$$

thus $T: P C(J, \mathbb{R}) \rightarrow P C(J, \mathbb{R})$.

Lemma 2.1 Since $W_{1}(t, s), W_{2}(t, s)$ in (3) and (4), when $t, s \in[0,1]$,

$$
\left|W_{1}(t, s)\right|<2(m+1),\left|W_{2}(t, s)\right|<2 m .
$$

Proof. When $0 \leq t \leq t_{1}, 0 \leq s \leq 1$,

$$
\begin{gathered}
\left|W_{1}(t, s)\right| \leq|\chi(0, t, s)|+\left|\frac{b}{(a+b)} \sum_{i=1}^{m+1}\right| \chi\left(s_{i-1}, t_{i}, s\right) \mid<2(m+1) . \\
\left|W_{2}(t, s)\right| \leq\left|\frac{b}{(a+b)}\right| \sum_{i=1}^{m}\left|\chi\left(t_{i}, s_{i}, s\right)\right| \leq m<2 m .
\end{gathered}
$$

When $s_{k}<t \leq t_{k+1}, 0 \leq s \leq 1, k=1,2, \ldots, m$,

$$
\begin{gathered}
\left|W_{1}(t, s)\right| \leq \sum_{i=1}^{k}\left|\chi\left(t_{i}, s_{i}, s\right)\right|+\left|\frac{b}{(a+b)}\right| \sum_{i=1}^{m}\left|\chi\left(t_{i}, s_{i}, s\right)\right|<2(m+1) \\
\left|W_{2}(t, s)\right| \leq \sum_{i=1}^{k}\left|\chi\left(t_{i}, s_{i}, s\right)\right|+\left|\frac{b}{(a+b)} \sum_{i=1}^{m}\right| \chi\left(t_{i}, s_{i}, s\right) \mid \leq k+m<2 m
\end{gathered}
$$

When $t_{k}<t \leq s_{k}, 0 \leq s \leq 1, k=1,2, \ldots, m$,

$$
\begin{gathered}
\left|W_{1}(t, s)\right| \leq \sum_{i=1}^{k}\left|\chi\left(s_{i-1}, t_{i}, s\right)\right|+\left|\frac{b}{(a+b)}\right| \sum_{i=1}^{m+1}\left|\chi\left(s_{i-1}, t_{i}, s\right)\right|<2(m+1), \\
\left|W_{2}(t, s)\right| \leq\left|\chi\left(t_{k}, t, s\right)\right|+\sum_{i=1}^{k-1}\left|\chi\left(t_{i}, s_{i}, s\right)\right|+\left|\frac{b}{(a+b)}\right| \sum_{i=1}^{m}\left|\chi\left(t_{i}, s_{i}, s\right)\right| \leq 1+k-1+m<2 m .
\end{gathered}
$$

Above all, when $t, s \in[0,1],\left|W_{1}(t, s)\right|<2(m+1),\left|W_{2}(t, s)\right|<2 m$.

Lemma 2.2 The operator $T: P C(J, \mathbb{R}) \rightarrow P C(J, \mathbb{R})$ is completely continuous.

Proof. Firstly, we proof that $T$ is an continuous operator.

Let $\quad u_{n}, u \in P C(J, \mathbb{R}), n=1,2, \ldots \quad, \quad$ and $\quad\left\|u_{n}-u\right\|_{P C} \rightarrow 0(n \rightarrow \infty) \quad$, For any $t \in J \quad$ we have $u_{n}(t) \rightarrow u(t)(n \rightarrow \infty)$, Since $f, g, M_{k}, Q_{k}$ are continuous, from Lebesgue control convergence theorem we have

$$
\begin{gathered}
\int_{0}^{1}\left|f\left(s, u_{n}(s)\right)-f(s, u(s))\right| \mathrm{d} s \rightarrow 0, \int_{0}^{1}\left|g\left(s, u_{n}(s)\right)-g(s, u(s))\right| \mathrm{d} s \rightarrow 0,(n \rightarrow \infty) . \\
\left|M_{k}\left(s, u_{n}(s)\right)-M_{k}(s, u(s))\right| \rightarrow 0, k=1,2, \ldots, m, n \rightarrow \infty . \\
\left|Q_{k}\left(s, u_{n}(s)\right)-Q_{k}(s, u(s))\right| \rightarrow 0, k=1,2, \ldots, m, n \rightarrow \infty .
\end{gathered}
$$

we have $\left\|T u_{n}-T u\right\|_{P C} \rightarrow 0,(n \rightarrow \infty)$, thus $T$ is continuous.

Secondly, we proof that $T$ is a compact operator. 
Let $\quad B_{r}=\{u \in P C(J, \mathbb{R}):\|u\| \leq r\} \quad, \quad M_{f}=\max _{(t, u) \in[0,1] \times[-r, r]}|f(t, u)| \quad, \quad M_{g}=\max _{(t, u) \in[0,1] \times[-r, r]}|g(t, u)| \quad$, $M_{M}=\max _{1 \leq k \leq m, u \in[-r, r]}\left|M_{k}\left(t_{k}, u\right)\right|, M_{Q}=\max _{1 \leq k \leq m, u \in[-r, r]}\left|Q_{k}\left(t_{k}, u\right)\right|$. For any $u \in B_{r}$, since Lemma 2.1 we have

$$
\begin{aligned}
|T u(t)| & =|A u(t)+B u(t)+G u(t)| \\
& \leq \int_{0}^{1}\left|W_{1}(t, s) f(s, u(s))\right| \mathrm{d} s+\int_{0}^{1}\left|W_{2}(t, s) g(s, u(s))\right| \mathrm{d} s+|G u(t)| \\
& \leq 2(m+1) M_{f}+2 m M_{g}+2 m\left(M_{Q}+M_{M}\right) .
\end{aligned}
$$

Thus $T\left(B_{r}\right)$ is uniformly bounded. It's obvious that when $t \in\left[0, t_{1}\right], \quad\left(s_{k}, t_{k+1}\right], k=1,2, \ldots, m-1$, $\left(t_{k}, s_{k}\right], k=1,2, \ldots, m,\left(s_{m}, 1\right], T\left(B_{r}\right)$ is isocratic, From Arzela-Ascoli theorem we have $T$ is a compact operator.

Above all, we have $T$ is a completely operator.

\section{Existence and Uniqueness of Solutions to Boundary Value Problems}

Let

(H1) There are non-negative real numbers $a_{0}, a_{1}, b_{0}, b_{1}, p_{0}, p_{1}, l_{0}, l_{1}$, constants $\sigma, \theta, \gamma, \eta>0$, for $t \in J$ and any $u \in \mathbb{R}$,

$$
|f(t, x)| \leq a_{0}+a_{1}|u|^{\sigma},|g(t, u)| \leq b_{0}+b_{1}|u|^{\theta},\left|M_{k}(t, u)\right| \leq p_{0}+p_{1}|u|^{\gamma},\left|Q_{k}(t, u)\right| \leq l_{0}+l_{1}|u|^{\eta} .
$$

(H2)There are constants $L_{1}, L_{2} \geq 0$, for any $t \in J$ and $u_{1}, u_{2} \in \mathbb{R}$ we have

$$
\begin{gathered}
\left|f\left(t, u_{1}\right)-f\left(t, u_{2}\right)\right| \leq L_{1}\left|u_{1}-u_{2}\right|,\left|g\left(t, u_{1}\right)-g\left(t, u_{2}\right)\right| \leq L_{2}\left|u_{1}-u_{2}\right| \\
\left|M_{k}\left(t, u_{1}\right)-M_{k}\left(t, u_{2}\right)\right| \leq L_{3}\left|u_{1}-u_{2}\right|,\left|Q_{k}\left(t, u_{1}\right)-Q_{k}\left(t, u_{2}\right)\right| \leq L_{4}\left|u_{1}-u_{2}\right| .
\end{gathered}
$$

Lemma 3.1 If (H1) and $0<\sigma, \theta, \gamma<1$, we have at least one solution in problem (1).

Proof. Let

$$
\begin{gathered}
r_{1} \geq \max \left\{1,10 m\left(a_{0}+b_{0}+p_{0}+l_{0}\right),\left(10(m+1) a_{1}\right)^{\frac{1}{1-\sigma}},\left(10 m b_{1}\right)^{\frac{1}{1-\theta}},\left(10 m p_{1}\right)^{\frac{1}{1-\gamma}},\left(10 m p_{1}\right)^{\frac{1}{1-\eta}}\right\}, \\
D=\left\{u \in P C(J, \mathbb{R}):\|u\|_{P C} \leq r_{1}\right\},
\end{gathered}
$$

thus we have $D$ as a non-empty bounded closed convex set in $P C(J, \mathbb{R})$.

For any $u \in D$ we have

$$
\begin{aligned}
|T u(t)|= & |A u(t)+B u(t)+G u(t)| \\
& \leq \int_{0}^{1}\left|W_{1}(t, s) f(s, u(s))\right| d s+\int_{0}^{1}\left|W_{2}(t, s) g(s, u(s))\right| d s+|G u(t)| \\
& <2(m+1)\left(a_{0}+a_{1} r_{1}^{\sigma}\right)+2 m\left(b_{0}+b_{1} r_{1}^{\theta}\right)+2 m\left(p_{0}+p_{1} r_{1}^{\gamma}\right)+2 m\left(l_{0}+l_{1} r_{1}^{\eta}\right) \\
& <2(m+1) a_{0}+2 m b_{0}+2 m p_{0}+2 m l_{0}+2(m+1) a_{1} r_{1}^{\sigma}+2 m b_{1} r_{1}^{\theta}+2 m p_{1} r_{1}^{\gamma}+2 m l_{1} r_{1}^{\eta} \\
& \leq r_{1}, \quad k=0,1, \cdots, m .
\end{aligned}
$$

Thus $\|T u\|_{P C} \leq r_{1}$ and $T(D) \subset D$. Since Lemma 2.4 we have $T$ is completely continious, since Schauder fixed point theorem we have at least one solution in problem (1).

Lemma 3.2 If (H1) and $\sigma=\theta=\gamma=1$ if $0<2(m+1) a_{1}+2 m b_{1}+2 m p_{1}+2 m l_{1}<1$, we have at least one solution in problem (1).

Proof. Let $r_{2} \geq \frac{2(m+1) a_{0}+2 m b_{0}+2 m p_{0}+2 m l_{0}}{1-\left(2(m+1) a_{1}+2 m b_{1}+2 m p_{1}+2 m l_{1}\right)}, E=\left\{u \in P C(J, \mathbb{R}):\|u\|_{P C} \leq r_{2}\right\}$, thus $E$ is a nonempty bounded closed convex set in $P C(J, \mathbb{R})$.

For any $u \in E$,we have

$$
|T u(t)|=|A u(t)+B u(t)+G u(t)|
$$




$$
\begin{aligned}
& \leq \int_{0}^{1}\left|W_{1}(t, s) f(s, u(s))\right| d s+\int_{0}^{1}\left|W_{2}(t, s) g(s, u(s))\right| d s+|G u(t)| \\
& <\frac{(m+2) a_{0}}{\Gamma(\alpha)}+\frac{2(m+1) b_{0}}{\Gamma(\beta)}+(m+1) l_{0}+\left(\frac{(m+2) a_{1}}{\Gamma(\alpha)}+\frac{2(m+1) b_{1}}{\Gamma(\beta)}+(m+1) l_{1}\right) r_{2} \\
& <2(m+1) a_{0}+2 m b_{0}+2 m p_{0}+2 m l_{0}+2(m+1) a_{1} r_{2}+2 m b_{1} r_{2}+2 m p_{1} r_{2}+2 m l_{1} r_{2} \\
& \leq r_{2}, \quad k=0,1, \cdots, m .
\end{aligned}
$$

thus $\|T u\|_{P C} \leq r_{2}, T(D) \subset D$. Since Lemma 2.4 we have $T$ is completely continious, since Schauder fixed point theorem we have at least one solution in problem $(1)$.

Lemma 3.3 If $(\mathrm{H} 2)$, and $0<2(m+1) L_{1}+2 m L_{2}+2 m L_{3}+2 m L_{4}<1$, Then the boundary value problem (1) has a unique solution on $P C(J, \mathbb{R})$.

Proof. For any $u_{1}, u_{2} \in P C(J, \mathbb{R})$ we have

$$
\begin{aligned}
& \left|T u_{1}(t)-T u_{2}(t)\right|=\left|A u_{1}(t)-A u_{2}(t)+B u_{1}(t)-B u_{2}(t)+G u_{1}(t)-G u_{2}(t)\right| \\
& \leq \int_{0}^{1}\left|W_{1}(t, s)\right|\left|f\left(s, u_{1}(s)\right)-f\left(s, u_{2}(s)\right)\right| d s+\int_{0}^{1}\left|W_{2}(t, s)\right| \mid g\left(s, u_{1}(s)\right)-g\left(s, u_{2}(s)|d s+| G u_{1}(t)-G u_{2}(t) \mid\right. \\
& <\left(2(m+1) L_{1}+2 m L_{2}+2 m L_{3}+2 m L_{4}\right)\left\|u_{1}-u_{2}\right\|_{P C} \\
& =\left(N_{0} L_{1}+N_{1} L_{2}+N_{2} L_{3}\right)\left\|u_{1}-u_{2}\right\|_{P C} .
\end{aligned}
$$

Since $0<2(m+1) L_{1}+2 m L_{2}+2 m L_{3}+2 m L_{4}<1$, we have $T$ is the compression map and the boundary value problem $(1)$ has a unique solution on $P C(J, \mathbb{R})$.

\section{References}

1. J. R. Wang Y, Zhou, and Z. Lin. "On a new class of impulsive fractional differential equations," Applied Mathematics and Computation, vol. 242, pp. 649-657, 2014.

2. S. Yu, "Optimal controls for a class of impulsive fractional differential equations with nonlocal conditions," Advances in Difference Equations, vol. 125, 2018.

3. J. R. Wang, K. Shah, and A. Ali. "Existence and Hyers-Ulam stability of fractional nonlinear impulsive switched coupled evolution equations," Mathematical Methods in the Applied Sciences, vol. 41, no.7, pp. 23922402, 2018.

4. D. Yang, J. R. Wang, and D. O'Regan, "A class of nonlinear non-instantaneous impulsive differential equations involving parameters and fractional order," Applied Mathematics and Computation, vol. 321, pp. 654-671, 2018.

5. K. Avadhesh, M. Muslim, and R. Sakthivel. "Controllability of the Second-Order Nonlinear Differential Equations with Non-instantaneous Impulses," Journal of Dynamical and Control Systems, vol. 24, pp. 325-342, 2017.

6. X. P. Liu, and M. Jia, "Existence of solutions for the integral boundary value problems of fractional order impulsive differential equations," Mathematical Methods in the Applied Sciences, vol. 39, pp. 475-487,2016.

7. J. V. D. C. Sousa, D. D. S. Oliveira, and E. C. D. Oliveira. "On the existence and stability for noninstantaneous impulsive fractional integrodifferential equation, " Mathematical Methods in the Applied Sciences, vol. 42, no. 4, pp. 1249-1261, 2019 .

8. M. Muslim, and A. Kumar, "Controllability of Fractional Differential Equation of Order $\alpha \in[1,2]$ with noninstantaneous Impulses," Asian Journal of Control, vol. 20, no. 2, pp. 935-942, 2018.

9. Z. Bo, "Periodic boundary value problems for fractional semilinear integro-differential equations with noninstantaneous impulses," Boundary Value Problems, vol. 128, 2018. 